\title{
A gyrofluid description of Alfvénic turbulence and its parallel electric field
}

\author{
N.H. Bian and E.P. Kontar \\ Department of Physics and Astronomy, \\ The University of Glasgow, G12 8QQ, United Kingdom
}

\begin{abstract}
Anisotropic Alfvénic fluctuations with $k_{\|} / k_{\perp} \ll 1$ remain at frequencies much smaller than the ion cyclotron frequency in the presence of a strong background magnetic field. Based on the simplest truncation of the electromagnetic gyrofluid equations in a homogeneous plasma, a model for the energy cascade produced by Alfvénic turbulence is constructed, which smoothly connect the large magnetohydrodynamics (MHD) scales and the small "kinetic" scales. Scaling relations are obtained for the electromagnetic fluctuations, as a function of $k_{\perp}$ and $k_{\|}$. Moreover, a particular attention is paid to the spectral structure of the parallel electric field which is produced by Alfvénic turbulence. The reason is the potential implication of this parallel electric field in turbulent acceleration and transport of particles. For electromagnetic turbulence, this issue was raised some time ago in [A. Hasegawa, K. Mima, J. Geophys. Res. 831117 (1978)].
\end{abstract}




\section{INTRODUCTION}

At large wave-numbers in the direction perpendicular to the ambient magnetic field, Alfvén waves produce a significant compression of the plasma which results in the creation of a parallel electric field via the thermo-electric effect. The parallel electric field associated with a spectrum of kinetic Alfvén waves leads to wave-particle interactions that can cause turbulent transport phenomena. This was pointed out already some time ago in Reference [1], in the context of the magnetospheric plasma. Starting from the drift-kinetics for the electrons, the authors of Ref.[1] derived a quasi-linear diffusion equation in both momentum and coordinate space, a so-called double-diffusion, showing the pivotal role played by the parallel electric field spectrum. In fact, their result is valid for any kind of low-frequency electromagnetic turbulence, but they used the properties of the kinetic Alfvén wave to give order of magnitude estimates of various transport coefficients (density, momentum) and the electrons heating rate. The solar wind is a natural laboratory plasma where the spectral properties of electromagnetic turbulence can be probed with good accuracy down to scales below the ion Larmor radius. Turbulent Alfvénic fluctuations observed in the solar wind have a solar origin. Hence, beside their intrinsic interests, solar wind measurements can also be used as a diagnostic tool to infer the properties of the turbulence closer to the sun, in the corona, where the plasma conditions are however different. For low-frequency Alfvénic fluctuations, the spectral energy density of the parallel electric field remains a small fraction of the electro-magnetic energy density. Hence, it is hardly a measurable quantity. However, since the parallel electric field is the actual force which mediates the wave-particle interaction, it is interesting to investigate its spectral structure in this range of spatio-temporal scales. This task is motivated by its potential relevance for turbulent acceleration and transport and is facilitated by more recent advances in the understanding of Alfvénic turbulence, mainly from theory and observations of the solar wind.

The starting point is a minimal fluid model of the non-linear dynamics of low-frequency kinetic Alfvén waves, also capable of capturing the effect associated with the thermal Larmor radius of the ions, $\rho_{i}=v_{T_{i}} / \omega_{c i}$ with $v_{T_{i}}=\sqrt{T_{i} / m_{i}}$ and $\omega_{c i}=e B_{0} / m_{i} c$. This model can be obtained as a truncation of the electromagnetic gyrofluid equations in a constant background magnetic field, by assuming a constant temperature for both the ions and the electrons, a constant background ion density and discarding all but the lowest two parallel 
moments of the electron kinetics (see [2-5] and references therein). These gyrofluid equations are moments of the gyrokinetic equations. Gyrokinetics owe its name from being based on an averaging of the kinetic and Maxwell equations over the gyromotion of the particles and, hence, it applies in the limit of frequencies small compared to the ion cyclotron frequency, $\omega \ll \omega_{c i}$. Extensive reviews on gyrokinetics can be found in Refs.[6-8]. Gyrokinetics and their fluid moments are valid in the limit of small Larmor radius, $\rho_{i} \ll L, L$ being the macroscopic length scale of the plasma, and small anisotropic fluctuations, in the sense that $k_{\|} / k_{\perp} \sim \delta B / B_{0} \ll 1, B_{0}$ being the magnitude of the background magnetic field. As for reduced-MHD [9 11], these ordering imply pressure balance in the direction perpendicular to the background magnetic field, such that the fast mode is ordered out. Moreover, because the gyroaverage procedure eliminates the cyclotron resonance, the only type of wave-particle interaction that remains possible is through the Landau resonance between the particles and the parallel electric force or the magnetic mirror one. On other central assumption of gyrokinetics, and hence of its fluid counterpart, is the small deviation of the distribution functions of the particles from a background distribution which is here a Maxwellian distribution.

The electromagnetic gyrofluid equations are presented first in Section II. Then they are used, in Section III, to construct a model of the energy cascade of Alfvénic turbulence from the large MHD scales down to the small kinetic scales. The spectral structure of the parallel electric field produced by Alfvénic turbulence is studied in Section IV. Finally, possible implications of the existence of this parallel electric field are discussed in Section V.

\section{THE ELECTROMAGNETIC GYROFLUID MODEL}

The gyrofluid model considered in this work involves the electron density $n_{e}$, the magnetic flux function $\psi=-A_{z}$, where $\mathbf{A}$ is the vector potential, and the electrostatic potential $\phi, z$ being the coordinate along the background field $\mathbf{B}_{0}[2-5]$. Adopting an MHD normalization (see the Appendix), these equations are

$$
\begin{gathered}
\partial_{t} n_{e}+\left[\phi, n_{e}\right]-\nabla_{\|} J=0, \\
\partial_{t} \psi+\nabla_{\|}\left(\rho_{s}^{2} n_{e}-\phi\right)=0 .
\end{gathered}
$$

The Poisson bracket is defined as $[f, g]=\mathbf{z} . \nabla f \times \nabla g$, where $\mathbf{z}$ is the unit vector along $\mathbf{B}_{0}$, and $\nabla_{\|} f=\partial_{z} f+[\psi, f]$ for any fields $f$ and $g$. The quantity $J=\nabla_{\perp}^{2} \psi$ is the parallel current 
density and $\rho_{s}=c_{s} / \omega_{c i} L$ is the normalized ion sound Larmor radius with $c_{s}=\sqrt{T_{e} / m_{i}}$. This system is closed by the gyrokinetic Poisson equation,

$$
n_{e}=\frac{\left(\Gamma_{0}-1\right)}{\rho_{i}^{2}} \phi
$$

where $\Gamma_{0}$ is an integral operator which describes the average of the electrostatic potential over a ring of Larmor radius $\rho_{i}$. In Fourier space, $\Gamma_{0}(b)$ simply becomes

$$
\Gamma_{0}(b)=e^{-b} I_{0}(b)
$$

where $b=\rho_{i}^{2} k_{\perp}^{2}$ and $I_{0}$ is the modified Bessel function of the first kind. The above equations conserve the total energy given by

$$
E=\int d \mathbf{x}\left[\left(\nabla_{\perp} \psi\right)^{2}+\rho_{s}^{2} n_{e}^{2}+\phi\left(1-\Gamma_{0}\right) \phi / \rho_{i}^{2}\right] .
$$

The model is valid for $v_{T i}^{2} / v_{A}^{2} \ll 1$, and moreover, since the effects associated with the electron inertia are not taken into account, it means that the conditions $v_{T e}^{2} / v_{A}^{2} \gg 1$ and $k_{\perp} d_{e} \ll 1$ must also be satisfied, $v_{A}$ being the Alfvén speed and $d_{e}$ the electron skin depth. In other words, the present model only describes the "kinetic" regime of the dispersive Alfvén wave, not its "inertial" regime.

The following comments are due. When a Pade approximant of the operator $\Gamma_{0}(b)$ is used, which gives :

$$
\Gamma_{0}(b)-1 \approx-\frac{b}{1+b},
$$

the Poisson equation is converted into:

$$
\left(1-\rho_{i}^{2} \nabla_{\perp}^{2}\right) n_{e}=\nabla_{\perp}^{2} \phi
$$

The model (1)-(3) with the Pade approximation (7), also including electron inertial effects, was studied in the context of collisionless magnetic reconnection in Ref.[5]. A well known effect of the electron inertia is the breaking of the frozen-in condition for the magnetic field.

When $k_{\perp} \rho_{i} \ll 1$, relation $(7)$ expresses the fact that the density is also equal to the plasma vorticity $n_{e}=\nabla_{\perp}^{2} \phi$, in which case Eqs.(1)-(2) become equivalent to a low- $\beta$ HallMHD model (see Appendix and e.g. Ref.[12]). Reduced Hall-MHD is an extension of the standard reduced MHD with the Hall and the electron pressure effect accounted for in the Ohms's law. The parallel component of the latter, i.e. Eq.(2), can also be written as

$$
E_{\|}=-\rho_{s}^{2} \nabla_{\|} n_{e}
$$


which shows the existence of a parallel electric field that arises from electron pressure gradient along the field lines. Electrons are here assumed to be isothermal. On the contrary, $E_{\|}=0$ is the standard reduced MHD Ohm's law. Equation (88) is a relation between the parallel electric field and the compressive density fluctuation, the parallel derivative being taken along the total magnetic field comprising the background plus its perpendicular perturbation. Implicit in Hall-MHD is the assumption of cold ions, or at least $\tau \equiv T_{i} / T_{e} \ll 1$. On the contrary, the gyrofluid model is valid for arbitrary value of $T_{i} / T_{e}$. An other simplification can be reached in the limit $k_{\perp} \rho_{i} \gg 1$, i.e. $n_{e}=-\phi / \rho_{i}^{2}$, in which case Eqs.(1)-(3) become similar to the reduced electron-MHD (see Appendix and Ref.[8]).

Assuming that all fields vary like $e^{i(\omega t-\mathbf{k} \cdot \mathbf{x})}$, the linearized equations (1)-(3) for the Fourier components are

$$
\begin{gathered}
\omega n_{e}-k_{\|} k_{\perp}^{2} \psi=0, \\
\omega \psi+k_{\|}\left(\phi-\rho_{s}^{2} n_{e}\right)=0, \\
n_{e}=\left(\Gamma_{0}(b)-1\right) \phi / \rho_{i}^{2} .
\end{gathered}
$$

Hence, this system yields the dispersion relation :

$$
\omega^{2}=k_{\|}^{2} \rho_{s}^{2} k_{\perp}^{2}\left(1-\frac{\tau}{\Gamma_{0}(b)-1}\right)
$$

with $\Gamma_{0}(b)=e^{-b} I_{0}(b)$. The Pade approximation for the operator $\Gamma_{0}(b)$ gives

$$
\omega^{2}=k_{\|}^{2}\left[1+k_{\perp}^{2}\left(\rho_{s}^{2}+\rho_{i}^{2}\right)\right]
$$

which is a standard expression for the frequency of the Alfvén wave in the "kinetic" regime[1].

Let us notice that the gyrofluid derivation of the the Alfvén wave frequency gives the same result as its kinetic counterpart, however the latter also provides the imaginary part, i.e. the coefficient associated with Landau damping. In the model proposed below for the energy cascade produced by Alfvénic turbulence, any form of dissipation, including this collisionless one, is neglected at all except the smallest scales where it is supposed to balance the energy injected at large scales. For Alfvénic perturbation, relations between the density, the potential and the flux function are provided by the equations (9)-(11), the magnitude of the perpendicular magnetic field fluctuation is given by $B_{\perp}=k_{\perp} \psi$, the magnitude of the perpendicular component of the electric field is given by $E_{\perp}=k_{\perp} \phi$ and the parallel component by $E_{\|}=\rho_{s}^{2} k_{\|} n_{e}$. 


\section{ANISOTROPIC ALFVÉNIC TURBULENCE}

For Alfvén waves, we observe from the energy integral Eq.(5), that the total energy density per wave-number in the perpendicular plane, i.e. the one dimensional energy spectrum denoted by $E_{k_{\perp}}$, is

$$
E_{k_{\perp}}=k_{\perp} \psi^{2}
$$

which coincides with the magnetic energy spectrum $E_{k_{\perp}}=\delta B_{\perp}^{2} / k_{\perp}$.

Locality of the non-linear interactions and constancy of the energy flux are assumed, hence the energy cascade rate $\epsilon$ is

$$
\epsilon=\frac{k_{\perp} E_{k_{\perp}}}{\tau_{N L}}
$$

On the other hand, the non-linear time scale is given by

$$
\tau_{N L}=\frac{1}{k_{\perp}^{2} \Phi}
$$

with the generalized stream-function $\Phi=\phi-\rho_{s}^{2} n_{e}$. Therefore, the non-linear time scale can also be written as a function of the magnetic perturbation, i.e.

$$
\tau_{N L}=\left[\rho_{s} k_{\perp}^{2}\left(1+\frac{\tau}{1-\Gamma_{0}}\right)^{1 / 2} k_{\perp} \psi\right]^{-1}
$$

with $k_{\perp} \psi=\left(E_{k_{\perp}} k_{\perp}\right)^{1 / 2}$. From (15), the energy spectrum is then obtained :

$$
E_{k_{\perp}} \propto \epsilon^{2 / 3} \rho_{s}^{-2 / 3} k_{\perp}^{-7 / 3}\left(1+\frac{\tau}{1-\Gamma_{0}}\right)^{-1 / 3}
$$

The Pade approximation for the latter expression gives

$$
E_{k_{\perp}} \propto \epsilon^{2 / 3} k_{\perp}^{-5 / 3}\left[1+k_{\perp}^{2}\left(\rho_{s}^{2}+\rho_{i}^{2}\right)\right]^{-1 / 3}
$$

When $T_{i} \ll T_{e}$, then $E_{k_{\perp}} \propto \epsilon^{2 / 3} k_{\perp}^{-5 / 3}\left(1+k_{\perp}^{2} \rho_{s}^{2}\right)^{-1 / 3}$ showing a breakpoint for $k_{\perp} \rho_{s} \sim 1$. Hence, this is an Hall-MHD result. On the contrary, when $T_{e} \ll T_{i}$, then $E_{k_{\perp}} \propto \epsilon^{2 / 3} k_{\perp}^{-5 / 3}(1+$ $\left.k_{\perp}^{2} \rho_{i}^{2}\right)^{-1 / 3}$ with breakpoint given by $k_{\perp} \rho_{i} \sim 1$. In general, the breakpoint occurs at $k_{\perp} \rho \sim 1$ with $\rho=\left(\rho_{s}^{2}+\rho_{i}^{2}\right)^{1 / 2}$. This breakpoint separates the MHD range with $E_{k_{\perp}} \propto \epsilon^{2 / 3} k_{\perp}^{-5 / 3}$ and the dispersive range with $E_{k_{\perp}} \propto \epsilon^{2 / 3} \rho^{-2 / 3} k_{\perp}^{-7 / 3}$.

Moreover, we have the following scaling relations: $B_{\perp} \propto \epsilon^{1 / 3} k_{\perp}^{-1 / 3}\left[1+k_{\perp}^{2}\left(\rho_{s}^{2}+\rho_{i}^{2}\right)\right]^{-1 / 6}$, $n_{e} \propto \epsilon^{1 / 3} k_{\perp}^{2 / 3}\left[1+k_{\perp}^{2}\left(\rho_{s}^{2}+\rho_{i}^{2}\right)\right]^{-2 / 3}, E_{\perp} \propto \epsilon^{1 / 3} k_{\perp}^{-1 / 3}\left[1+k_{\perp}^{2}\left(\rho_{s}^{2}+\rho_{i}^{2}\right)\right]^{-2 / 3}\left(1+k_{\perp}^{2} \rho_{i}^{2}\right)$. Notice that $n_{e}$ above refers to density fluctuations that arise from the kinetic Alfvén wave compression. 
The one dimensional energy spectrum for the perpendicular electric field is therefore $E_{\perp}^{2} / k_{\perp} \propto \epsilon^{2 / 3} k_{\perp}^{-5 / 3}\left(1+k_{\perp}^{2} \rho^{2}\right)^{-4 / 3}\left(1+k_{\perp}^{2} \rho_{i}^{2}\right)^{2}$. For $T_{i} \sim T_{e}$, the latter relation is a good fit to some solar wind measurements [8, 13]. It corresponds to a power index $-5 / 3$ in the MHD regime when $k_{\perp} \rho_{i} \ll 1$ and $-1 / 3$ in the dispersive range when $k_{\perp} \rho_{i} \gg 1$. When $T_{i} \gg T_{e}$, $E_{\perp} \propto \epsilon^{1 / 3} k_{\perp}^{-1 / 3}\left[1+k_{\perp}^{2} \rho_{i}^{2}\right]^{1 / 3}$, which has only one break-point at $k_{\perp} \rho_{i} \sim 1$. Notice however that in a plasma with $T_{e} \gg T_{i}$ then, $E_{\perp} \propto \epsilon^{1 / 3} k_{\perp}^{-1 / 3}\left[1+k_{\perp}^{2} \rho_{s}^{2}\right]^{-2 / 3}\left(1+k_{\perp}^{2} \rho_{i}^{2}\right)$, which has two break-points at $k_{\perp} \rho_{s} \sim 1$ and at $k_{\perp} \rho_{i} \sim 1$.

A theory for strong anisotropic Alfvén wave turbulence was developed by GoldreichSidrar 14]. According to this theory, the anisotropy of the turbulence is fixed by the condition,

$$
\omega \sim \tau_{N L}^{-1}
$$

which is a balance between the linear and the non-linear dynamical time scales. This leads to the following scale dependent anisotropy relation :

$$
k_{\|}\left(k_{\perp}\right) \sim \epsilon^{1 / 3} k_{\perp}^{2 / 3}\left[1+k_{\perp}^{2}\left(\rho_{s}^{2}+\rho_{i}^{2}\right)\right]^{-1 / 6} .
$$

This is also equivalent to the ordering relation

$$
\delta B_{\perp} \sim k_{\|} / k_{\perp} \ll 1
$$

with $\delta B_{\perp}\left(k_{\perp}\right)$ given above. We end this section by emphasizing that the scaling relations for the energy spectrum and anisotropy in the dispersive scales [8, 15] are similar to the ones of EMHD turbulence[16-19]

\section{PARALLEL ELECTRIC FIELD SPECTRUM}

Alfvénic turbulence involves electric field fluctuations $\mathbf{E}$ which possess a component parallel to the magnetic field $\mathbf{B}$. We now discuss the spectral structure of the parallel electric field produced by Alfvénic turbulence. The magnitude of the parallel electric field is small compared to the perpendicular one, hence hardly measurable and not expected to change the total electric field spectrum. Nevertheless, the parallel electric field can be important for particle acceleration and cross-field transport induced by Alfvénic turbulence, or in fact, by any type of electromagnetic turbulence. The physical reason is that the parallel electric field can efficiently accelerate particles along the magnetic field lines which are bent in the 
direction perpendicular to $\mathbf{B}_{0}$ due to the perturbation $\mathbf{B}_{\perp}$. Hence the correlation between acceleration and cross-field transport, and the pivotal role played by $E_{\|}$. The results of the previous sections provide the scaling of the parallel electric field fluctuation :

$$
E_{\|} \propto \epsilon^{1 / 3} \rho_{s}^{2} k_{\|} k_{\perp}^{2 / 3}\left[1+k_{\perp}^{2}\left(\rho_{s}^{2}+\rho_{i}^{2}\right)\right]^{-2 / 3}
$$

together with Eq.(21) for the relation $k_{\|}\left(k_{\perp}\right)$. It is therefore easy to verify that the magnitude of $E_{\|}$, as a function of $k_{\|}$, scales like

$$
E_{\|}\left(k_{\|}\right) \propto \rho_{s}^{2} k_{\|}^{2},
$$

when $k_{\|} \ll \epsilon^{1 / 3} \rho^{-2 / 3}$, and like

$$
E_{\|}\left(k_{\|}\right) \propto \epsilon k_{\|}^{-1} \frac{\rho_{s}^{2}}{\rho_{s}^{2}+\rho_{i}^{2}}
$$

when $k_{\|} \gg \epsilon^{1 / 3} \rho^{-2 / 3}$. Moreover, the magnitude of $E_{\|}$reaches its maximum,

$$
E_{\|} \sim \epsilon^{2 / 3} \frac{\rho_{s}^{2}}{\left(\rho_{s}^{2}+\rho_{i}^{2}\right)^{2 / 3}}
$$

at the breakpoint $k_{\|} \sim \epsilon^{1 / 3} \rho^{-2 / 3}$, which corresponds to to $k_{\perp} \rho \sim 1$, with $\rho=\left(\rho_{s}^{2}+\rho_{i}^{2}\right)^{1 / 2}$, where the two branches merge smoothly. It is clear that the condition $k_{\perp} \rho \gg 1$ does not have do be realized for $E_{\|}$to be of significant value because the latter reaches its maximum precisely at the boundary between the MHD and the "kinetic" scales.

\section{DISCUSSION AND CONCLUSION}

Based on a simple truncation of the gyrofluid equations, we constructed a model for the energy cascade produced by Alfvénic turbulence which smoothly connect the large magnetohydrodynamics (MHD) scales and the small "kinetic" scales. A similar model, with an emphasize on the effect of linear collisionless dissipation was proposed in Ref.[20]. Scaling relations are obtained for the electromagnetic fluctuations as a function of $k_{\perp}$ and $k_{\|}$and particular attention is paid to the spectral structure of the parallel electric field produced by Alfvénic turbulence. The reason is that wave-particle interactions through the parallel electric force can produce anomalous transport in both velocity and coordinate space. A first principle understanding of such turbulent process is provided by quasi-linear theory applied to the gyro-averaged drift-kinetic equations. Within the framework of quasilinear 
theory, the resulting diffusion equation for, say the electron distribution function $f$ reads (see Ref.1)

$$
\frac{\partial f}{\partial t}=\sum_{k} \nabla\left[\left(\frac{e}{m_{e}}\right)^{2} \frac{\pi}{2} \delta\left(\omega-k_{\|} v_{\|}\right) E_{\|}^{2} \nabla f\right]
$$

with the operator $\nabla$ defined as

$$
\nabla=\nabla_{v_{\|}}+\frac{k_{\perp} v_{\|}}{\omega \omega_{c e}} \nabla_{x}
$$

All quantities here are dimensional, $v_{\|}$is the component of the electron velocity parallel to the magnetic field and $x$ is the coordinate transverse to $\mathbf{B}_{0}$. The same type of equation can be written for the ion distribution function. A major ingredient entering the quasilinear diffusion coefficients, is the spectrum of the parallel electric field, which has been studied above for strong and anisotropic Alfvénic turbulence. The parallel electric field produced by the turbulence results in Landau damping of the turbulent field. The effect of any scale dependent damping process, can be accounted for on the energy cascade by writing a transport equation through wavenumber space as follow (see Ref.[20]):

$$
\frac{\partial E_{k_{\perp}}}{\partial t}+\frac{\partial}{\partial k_{\perp}}\left[\frac{k_{\perp}}{\tau_{N L}} E_{k_{\perp}}\right]=-2 \gamma E_{k_{\perp}}
$$

with $\gamma\left(k_{\perp}, k_{\|}\right)$, representing the damping rate. Recently, the effect of Landau damping on the energy spectrum was studied this way, based on linear gyrokinetic calculations [20] of the damping rate. However, the modification of the distribution function due to wave-particle interactions was not addressed. A possible approach is the standard quasi-linear theory. Let us notice that, in order for the fluid treatment to be valid, the number of accelerated particles must remain small compared to the number of core particles. Modeling of such a turbulent acceleration mechanism through the parallel electric field spectrum of Alfvénic fluctuations, its impact on the particles distribution function and back-reaction on the turbulent energy cascade, is the subject of ongoing work.

\section{APPENDIX}

In this appendix we provide few additional details concerning the gyrofluid model. We also discuss some of its limiting cases (RMHD, ERMHD) and the corresponding model energy spectra. The normalized equations (1)-(2) follow from the electron continuity and 
the parallel momentum equations, which can be written as

$$
\begin{gathered}
\partial_{t} \frac{n_{e}}{n_{0}}+\frac{c}{B_{0}}\left[\phi, \frac{n_{e}}{n_{0}}\right]=\partial_{z} v_{e z}+\frac{1}{B_{0}}\left[A_{z}, v_{e z}\right] \\
\partial_{t} A_{z}+\frac{c}{B_{0}}\left[\phi, A_{z}\right]=-c \partial_{z} \phi+\frac{c T_{e}}{e} \partial_{z} \frac{n_{e}}{n_{0}}+\frac{c T_{e}}{e B_{0}}\left[\frac{n_{e}}{n_{0}}, A_{z}\right]
\end{gathered}
$$

with $v_{e z}=\left(c / 4 \pi n_{0}\right) \nabla_{\perp}^{2} A_{z}$. This system is closed by the gyrokinetic Poisson equation

$$
n_{e}=\left(\Gamma_{0}-1\right) \frac{e n_{0}}{T_{i}} \phi
$$

Adopting the following "MHD" normalization,

$$
\left(\widehat{t}, \widehat{x}, \widehat{\phi}, \widehat{A}_{z}, \widehat{n}_{e}\right)=\left(\frac{t}{\tau_{A}}, \frac{x}{L}, \frac{\phi c}{L v_{A} B_{0}}, \frac{A_{z}}{L B_{0}}, \frac{n_{e} \omega_{c i} \tau_{A}}{n_{0}}\right),
$$

with $\tau_{A}=L / v_{A}$, then Eqs.(30)-(32) become Eqs.(1)-(3). Hence, there are two nondimensional parameters in this gyrofluid model : say, $\rho_{s}$ and $\rho_{i}$. When $k_{\perp} \rho_{i} \ll 1$, the Poisson equation gives $n_{e}=\nabla_{\perp}^{2} \phi$, and therefore Eqs.(1)-(2) are equivalent to the following low- $\beta$ Hall-MHD model :

$$
\begin{gathered}
\partial_{t} \nabla_{\perp}^{2} \phi+\left[\phi, \nabla_{\perp}^{2} \phi\right]-\nabla_{\|} J=0 \\
\partial_{t} \psi+\nabla_{\|}\left(\rho_{s}^{2} \nabla_{\perp}^{2} \phi-\phi\right)=0 .
\end{gathered}
$$

In the MHD approximation, $E_{\|}=0$ and therefore the standard RMHD reads :

$$
\begin{gathered}
\partial_{t} \nabla_{\perp}^{2} \phi+\left[\phi, \nabla_{\perp}^{2} \phi\right]-\nabla_{\|} J=0, \\
\partial_{t} \psi-\nabla_{\|} \phi=0
\end{gathered}
$$

Notice that the RMHD system is free of any non-dimensional parameter under the present normalization. The RMHD conserves the energy :

$$
E=\int d \mathbf{x}\left[\left(\nabla_{\perp} \psi\right)^{2}+\left(\nabla_{\perp} \phi\right)^{2}\right]
$$

Linearizing the RMHD system provides the frequency of the Alfvén wave $\omega= \pm k_{\|}$. Moreover, since the relation $\phi= \pm \psi$ holds for Alfvénic fluctuations, it follows from the energy integral that the one-dimensional energy spectrum is

$$
E_{k_{\perp}}=k_{\perp} \psi^{2}
$$


which coincides with the magnetic energy spectrum, i.e. $E_{k_{\perp}}=\delta B_{\perp}^{2} / k_{\perp}$ since $\delta B_{\perp}=k_{\perp} \psi$. Assuming locality of the non-linear interactions and constancy of the energy flux, the energy cascade rate $\epsilon$ is

$$
\epsilon=\frac{k_{\perp} E_{k_{\perp}}}{\tau_{N L}}
$$

On the other hand, the non-linear time scale is given by

$$
\tau_{N L}=\frac{1}{k_{\perp}^{2} \phi}
$$

Hence, this time scale can also be written in term of the magnitude of the magnetic fluctuation,

$$
\tau_{N L}=\frac{1}{k_{\perp}^{2} \psi},
$$

with $k_{\perp} \psi=\left(E_{k_{\perp}} k_{\perp}\right)^{1 / 2}$. The expression for the energy spectrum in the MHD regime is then obtained :

$$
E_{k_{\perp}} \propto \epsilon^{2 / 3} k_{\perp}^{-5 / 3}
$$

An other limit consists in taking $k_{\perp} \rho_{i} \gg 1$, in which case the Poisson equation (3) gives $\phi=-\rho_{i}^{2} n_{e}$, and hence Eqs.(1)-(2) become

$$
\begin{gathered}
\partial_{t} \phi+\rho_{i}^{2} \nabla_{\|} J=0, \\
\partial_{t} \psi-\left(\frac{\tau}{1+\tau}\right) \nabla_{\|} \phi=0 .
\end{gathered}
$$

An extension of this Electron-RMHD model, valid for a wider range of values of $v_{T i}^{2} / v_{A}^{2}$, was derived and studied previously in Ref.[8]. This ERMHD conserves the energy :

$$
E=\int d \mathbf{x}\left[\left(\nabla_{\perp} \psi\right)^{2}+\left(\frac{\tau+1}{\tau}\right) \frac{\phi^{2}}{\rho_{i}^{2}}\right] .
$$

Linearizing the ERMHD system provides the frequency of the dispersive kinetic Alfvén wave $\omega= \pm k_{\|} k_{\perp} \sqrt{\tau / 1+\tau} \rho_{i}$. Moreover, since the relation $\sqrt{\tau / 1+\tau}\left(\phi / \rho_{i}\right)= \pm k_{\perp} \psi$ holds for kinetic Alfvén waves, it is easily verified that the one-dimensional energy spectrum is again the magnetic energy spectrum $E_{k_{\perp}}=k_{\perp} \psi^{2}$. The non-linear decorrelation time scale is given by

$$
\tau_{N L}=\frac{1+\tau}{\tau} \frac{1}{k_{\perp}^{2} \phi}
$$

(see Ref.[8]). This is indeed the $k_{\perp} \rho_{i} \gg 1$ limit of the relation (16), since $n_{e}=-\phi / \rho_{i}^{2}$ also in this limit. Therefore, the expression for the energy spectrum in the dispersive range is

$$
E_{k_{\perp}} \propto \epsilon^{2 / 3} \rho_{i}^{-2 / 3}\left(\frac{1+\tau}{\tau}\right)^{1 / 3} k_{\perp}^{-7 / 3}
$$


[1] A. Hasegawa, K. Mima, J. Geophys. Res. 831117 (1978).

[2] F.L. Waelbroeck, R.D Hazeltine, P.J. Morrison, Phys. Plasmas 16032109 (2009).

[3] P.B. Snyder, G.W. Hammett, Phys. Plasmas 8, 3199 (2001).

[4] N.F. Loureiro, G.W. Hammett, J. Comput. Phys. 2274518 (2008).

[5] D. Grasso, F. Califano, F. Pegoraro, F. Porcelli, Plasma Physics Reports 26548 (2000).

[6] A.J. Brizard, T.S. Hahm, Rev. Mod. Phys. 79421 (2007).

[7] G. G. Howes, S. C. Cowley, W. D. Dorland, G. W. Hammett, E. Quataert, and A. A. Schekochihin, Astrophys. J. 651, 590 (2006).

[8] A.A. Schekochihin, S.C. Cowley, W. Dorland, G.W. Hammett, G.G. Howes, E. Quataert, T. Tatsuno, Astrophys. J. 182310 (2009).

[9] B.B. Kadomtsev, O.P. Pogutse, Sov. Phys. JETP 38, 283 (1974).

[10] H.R. Strauss, Phys. Fluids 19, 134 (1976).

[11] R.D. Hazeltine, M. Kotschenreuther, P.J. Morrison, Phys. Fluids 282466 (1985).

[12] N.H. Bian, D. Tsiklauri, Phys. Plasmas 16064503 (2009).

[13] S.D. Bale, P.J. Kellogg, F.S. Mozer, T.S. Horbury, H. Reme, Phys. Rev. Lett., 94, 215002 (2005).

[14] P. Goldreich, S. Sridhar, Astrophys. J. 438763 (1995).

[15] S.R. Cranmer, A.A. Van Ballegooijen, Astrophys. J. 594573 (2003).

[16] D. Biskamp, E. Schwarz, A. Zeiler, A. Celani, J.F. Drake, Phys. Plasmas 6751 (1999).

[17] C.S. Ng, A. Bhattacharjee, K. Germaschewski, S. Galtier, Phys. Plasmas 10, 1954 (2003).

[18] J. Cho, A. Lazarian, Astrophys. J. 615 L41 (2004).

[19] J. Cho, A. Lazarian, Astrophys. J. 701236 (2009).

[20] G.G Howes, S.C. Cowley, W. Dorland, G.W. Hammett, E. Quataert, A.A. Schekochihin, J. Geophys. Res. 113 A05103 (2008). 